\title{
Fingerprint analysis and pharmacological evaluation of Ailanthus altissima
}

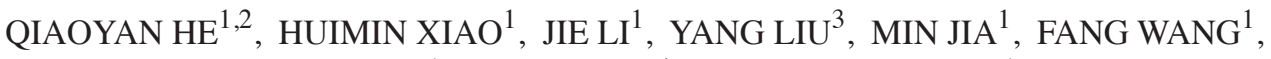 \\ YA ZHANG $^{1}$, WEN WANG ${ }^{4}$ and SIWANG WANG ${ }^{1}$ \\ ${ }^{1}$ Department of Natural Medicine, School of Pharmacy, The Fourth Military Medical University, Xi'an, Shaanxi 710032; \\ ${ }^{2}$ Department of Pharmacology, School of Pharmacy, Shanxi Medical College for Continuing Education, \\ Tai Yuan, Shanxi 030000; ${ }^{3}$ School of Pharmacy, Shaanxi University of Chinese Medicine, Xianyang, Shaanxi 712000; \\ ${ }^{4}$ Department of Traditional Chinese Medicine, Xijing Hospital of \\ The Fourth Military Medical University, Xi'an, Shaanxi 710032, P.R. China
}

Received October 19, 2016; Accepted February 8, 2018

DOI: $10.3892 / \mathrm{ijmm} .2018 .3492$

\begin{abstract}
Ailanthus altissima (AA) has been used in various anticancer prescriptions and showed excellent therapeutic effect. However, there is no report on the method of quality control and the anti-glioblastoma activity. In this study, we used a combinative method approach consisting of chromatographic fingerprinting and quantitative methods to analyze quality of different samples. The anti-glioblastoma activity and the possible mechanisms were studied by pharmacological methods. The samples were separated on a Kromasil 100-5 C18 column and the flow rate was $0.8 \mathrm{ml} / \mathrm{min}$ at $25^{\circ} \mathrm{C}$. The mobile phase was composed of $1 \%$ formic acid and $1 \%$ methanol-water at a flow rate of $0.8 \mathrm{ml} / \mathrm{min}$. The method was validated and applied to the quantification of different samples. Ten batches of AA from different provinces in China were detected, and we found that the contents of Ailanthone (AT) ranged from $0.21-1.78 \mathrm{mg} / \mathrm{g}$ and the relative retention times were similar in different origins. Ten batches of AA were analyzed by the high-performance liquid chromatography (HPLC) fingerprinting method and 19 common peaks were detected. The similarity of 10 batches is $<1.5 \%$. The peak areas in different samples were significantly different (0.682-0.954). We also found that AA induced oxidative stress first in U87 cells, then induced ER stress, finally activated the caspases which caused cell apoptosis. In conclusion, a method combining chromatographic fingerprinting and quantitative analysis can be used to control the quality of AA. AA could be used as a medicine or a constituent part of herb prescription to treat glioblastoma.
\end{abstract}

Correspondence to: Professor Siwang Wang, Department of Natural Medicine, School of Pharmacy, The Fourth Military Medical University, 169 West Changle Road, Xi'an, Shaanxi 710032, P.R. China E-mail:wangsiw@fmmu.edu.cn

Key words: Ailanthus altissima, fingerprint, glioblastoma, quality control, apoptosis

\section{Introduction}

Traditional Chinese Medicines (TCMs), especially herbal medicines, have been used as the main approaches in treating various diseases in China for $>2000$ years. TCMs are gaining increasing attention worldwide for their thousands of years of practice and the potential therapeutic applications. To obtain synergistic effects or diminish potential adverse effects, TCMs are commonly prescribed in combination (1). To our knowledge, the synergistic and therapeutic effects of TCMs are based on the joint contribution of multi-components (2). Generally, due to differences in cultivation areas, practices, plant origins, climate conditions and processing protocols among others, the chemical composition of herbal formulations may vary in a larger scale (3-5). Because of the multicomponent and multitarget nature of therapy, it is difficult to control the quality of the TCM preparation effectively. Thus, establishing an effective and feasible method which can control the quality of individual herbs or multiple herbs is necessary.

Ailanthus altissima Swingle (Simaroubaceae, AA), the tree-of-heaven, is native to China and was introduced to Europe around the end of 18th century. AA has been used in Chinese traditional medicine to treat various diseases including epilepsy, asthma, diarrhea, dysentery, heat ailments, and also as an astringent (6). Various biological activities has been observed in the stem bark of AA, including anti-plasmodial, anti-viral, anti-proliferative, cytotoxic and anti-malarial $(7,8)$. It has been reported that AA water decoction decreased the production of inflammatory cytokines including interleukin-6 (IL-6), tumor necrosis factor and IL-8, and the expression of nuclear factor- $\kappa \mathrm{B}$ in HMC-1 human mast cell line (9). Further study also showed that the EtOH extract of AA inhibited the generation of the cyclooxygenase-2 (COX-2) in BMMC cells (10). In previous phytochemical studies, quassinoids, indole alkaloids, lipids, fatty acids, phenolic derivatives, and volatile compounds from AA have been characterized (11-14). Extracts of AA and some isolated chemical compounds have shown some medicinal properties (15). Ailanthone (AT), one of the 
primary potent quassinoids in AA, has various biological activities, including anti-malarial, anti-inflammatory, anti-allergic, anti-HIV, antiulcer and antimicrobial activities (16-18). It inhibited growth of several cancer cell lines, including Jurkat, Hep3B, HepG2, R-HepG2, MCF-7, HeLa and A549 cells in vitro (17-20). However, details of their effect in inhibiting brain glioma growth is scarce.

There have been limited reports describing assays for simultaneous determination of the active components in AA. Only a few reports that separated some quassinoids from AA by high-performance liquid chromatography (HPLC) methods $(21,22)$, but only one study reported the determination of AT in soil, which was not suitable for biological analysis (23). A highly sensitive LC-MS/MS method has been used to analyse AT levels in rats plasma, however, these procedures require specialized equipment and are not suitable for chemical systems. For routine analysis in the production process of AA and its preparations, a quick and simple HPLC analytical method would be highly desirable. Here, a simple and feasible method for the quantitative determination of the active components in AA was developed to improve the QC. Cellular pharmacology experiments were also undertaken to assess the effect of different AA samples.

\section{Materials and methods}

Chemicals and herbal materials. The reference standard for AT was purchased from National Institute for the Control of Pharmaceutical and Biological Products (NICPP, China). The purity was determined to be $>98 \%$ based on a peak area normalization method by HPLC analysis. HPLC-grade acetonitrile and methanol were obtained from Burdick \& Jackson (Muskegon, MI, USA). Formic acid was purchased from Beijing Chemical Factory (Beijing, China). Purified water was prepared from a Millipore water purification system (Millipore, Billerica, MA, USA). Other reagents were from Sigma-Aldrich (St. Louis, MO, USA) and analytical grade.

Crude plant materials (lot no. S1-S10) were collected from ten regions (Henan, Shanxi, Anhui, Hebei, Guizhou, Guangxi, Sichuan, Yunnan, Zhejiang, Jiangsu, respectively) and met the requirements of Beijing Food and Drug Administration. All herbal medicines were identified by Professor Zhishu Tang of the School of Pharmacy, Shaanxi University of Chinese Medicine and voucher specimens were deposited in the Institute of Materia Medica, School of Pharmacy, the Fourth Military Medical University.

Preparation of the extracts. The stem bark of AA was air-dried and powdered. Each powdered sample was precisely weighed $(1 \mathrm{~kg})$ and extracted with $75 \% \mathrm{EtOH}$ three times at room temperature, and then the solution was evaporated in vacuo. For further use, all extracts were stored at $4^{\circ} \mathrm{C}$. Extracts were diluted if necessary.

Chromatographic conditions. HPLC analysis was performed on a Shimadzu LC-2010A HT HPLC equipped with a UV detector. The analytes were separated on a Kromasil 100-5-C18 column $(250 \times 4.6 \mathrm{~mm}, 5 \mu \mathrm{m})$. The mobile phase consisted of A (1\% acetonitrile) and B (water containing $1 \%$ formic acid). The linear gradient conditions were optimized as follows:
0-40 $\min , 3-20 \% \mathrm{~A} ; 40-45 \mathrm{~min}, 20-25 \% \mathrm{~A} ; 45-50 \mathrm{~min}$, 25-30\% A; 50-60 min, 30-60\% A; 60-65 min, 60-65\% A. The flow rate was $0.8 \mathrm{ml} / \mathrm{min}$ at $25^{\circ} \mathrm{C}$. The UV detection wavelength was set at $250 \mathrm{~nm}$ and sample injection volume was $10 \mu 1$.

Preparation of stock and working solutions. Stock solutions were prepared by dissolving accurate amounts of AA and AT in water, and stored at $4^{\circ} \mathrm{C}$. Then the stock standard solution was serially diluted with water in order to prepare calibration standard solutions of $0.825,0.412,0.206,0.103,0.052,0.026$, 0.013 and $0.0065 \mathrm{mg} / \mathrm{ml}$, respectively. For establishing the calibration curves, $10 \mu \mathrm{l}$ of the above working solutions were used. For preparing the calibration curve, the peak area of the analyte was taken as the Y-axis and the concentration of AT (mg/ml) was taken as the $\mathrm{X}$-axis.

Method validation. According to the guidelines of the China Food and Drug Administration (CFDA), HPLC fingerprinting analysis was developed and its stability, precision, and repeatability were validated. To evaluate sample stability, the same sample AA-S1 solution at different time-points $(0,0.5,1,2,4$, $6,8,12$ and $24 \mathrm{~h}$ ) were analyzed. Five replicates of a sample of an AA-S1 solution were performed to determine the precision on the same day. To confirm the repeatability of results, five different sample solutions prepared from the same sample (AA-S1) were analyzed. To measure the relative recoveries, six concentrations $(11.0108,11.0160,6.0012,6.0511,2.8875$ and $2.9438 \mathrm{mg}$ ) of AT were added into the same sample (AA-S1). Five replicates of the resultant samples were analyzed with the HPLC method established above. The measured to the added amount ratio was used to calculate the recovery. The LOD and LOQ were determined from the $\mathrm{S} / \mathrm{N}$ level of three and ten, respectively.

Pharmacological studies. The human brain glioma cell line U87 was obtained from the American Type Culture Collection (Rockville, MD, USA). Cells were cultured in Dulbecco's modified essential medium (DMEM) supplemented with $10 \%$ fetal bovine serum (FBS), and penicillin/streptomycin (Gibco, Carlsbad, CA, USA) in an atmosphere of $5 \% \mathrm{CO}_{2}$ at $37^{\circ} \mathrm{C}$. For drug treatments, the U87 cell lines were treated with the vehicle DMEM or various concentrations of AA for 1-3 days. For some experiments, the AA-treated cells were also incubated with or without $20 \mu \mathrm{M}$ of Z-VAD-fmk, a broad-spectrum caspase inhibitor, or the antioxidant reagent, $\mathrm{N}$-acetyl cysteine (NAC, $2.5 \mathrm{mM}$ ). After different treatments, cell survival rate was measured by MTT. ROS and MDA levels were measured by corresponding kits according to the instructions.

To measure protein expression, U87 cells were collected and dissolved in lysis buffer containing protease inhibitors (Sigma-Aldrich), and then centrifuged at 10,000 rpm for $20 \mathrm{~min}$. The supernatant was collected for protein analysis and stored at $-80^{\circ} \mathrm{C}$ until used. Thirty micrograms of the cell lysates was separated by using $10 \%$ sodium dodecyl sulfatepolyacrylamide gel electrophoresis (SDS-PAGE). Then, transferred to polyvinylidene fluoride membrane (PVDF), and immunoblotted with antibodies against cleaved-caspase-8, cleaved-caspase-3, p-PERK, PERK, elF2 $\alpha$, p-elF2 $\alpha$, Bax, 

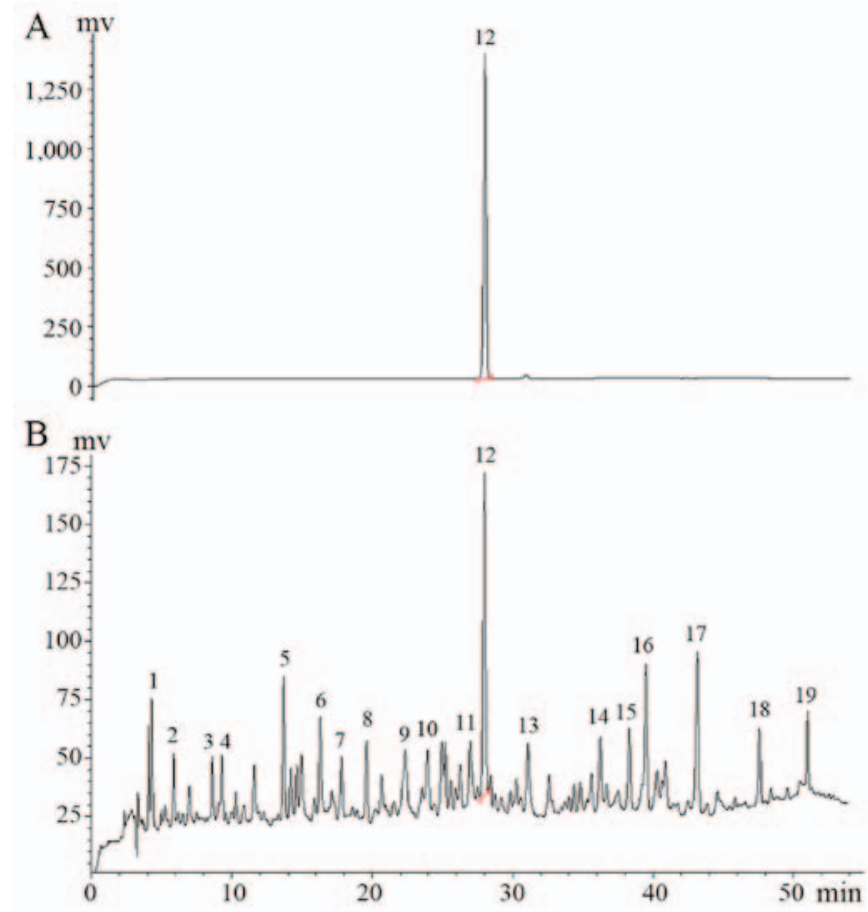

Figure 1. Representative high-performance liquid chromatography (HPLC) chromatograms of (A) Ailanthone (AT) and (B) Ailanthus altissima (AA).

Bcl-2 and $\beta$-actin (Santa Cruz Biotechnology, Inc., Dallas, TX, USA). Bands were visualized using the ECL Plus detection system (GE Healthcare, Freiburg, Germany). Densitometric analyses were performed using Image-Pro Plus.

Data analysis. The data analysis of chromatographic fingerprinting was conducted by Similarity Evaluation System for Chromatographic Fingerprint of Traditional Chinese Medicine software (version 2008), which was recommended by the State Food and Drug Administration of China. One AA sample from one origin was chosen and evaluated through the proposed pharmacological method. Data are expressed as the mean \pm standard deviation of triplicate measurements. The comparison was done using the Student's t-test. $\mathrm{P}<0.05$ was considered significant.

\section{Results}

Optimization of chromatographic conditions. To determine the best separation mechanism and to obtain as much chemical information as possible in chromatograms, different HPLC parameters including different columns, detection wavelengths, mobile phases, and gradient elution conditions were examined and compared. Yilite Hypersil base deactivated silica C18 column, Yilite SinoChrom ODS-BP $\mathrm{C}_{18}$ column, and Kromasil 100-5 C18 column were investigated and compared. Kromasil 100-5 C18 column had good peak separation and sharp peaks, so Kromasil 100-5 C18 column was chosen in the further study. The effect of mobile phase composition (acetonitrile-water and methanol-water together with different organic solvents including formic acid, acetic acid, triethylamine, and phosphoric acid) on chromatographic separation was investigated. Adding $1 \%$ formic acid in the
Table I. Calibration curve parameters, precision, repeatability, stability and recovery of AT.

\begin{tabular}{clc}
\hline No. & \multicolumn{1}{c}{ Item } & AT \\
\hline 1 & Calibration curves & $\mathrm{y}=2.2 \mathrm{x}-20.9$ \\
2 & Linear range $(\mu \mathrm{g} / \mathrm{ml})$ & $6.44-825 \mu \mathrm{g} / \mathrm{ml}$ \\
3 & Correlation coefficient, $\mathrm{r}^{2}$ & 0.9992 \\
4 & Precision RSD $\%, \mathrm{n}=6$ & 2.1 \\
5 & Repeatablity RSD $\%, \mathrm{n}=6$ & 1.9 \\
6 & Stability RSD $, \%, \mathrm{n}=6$ & 2.2 \\
7 & Recovery, mean, RSD $, \%, \mathrm{n}=3$ & 1.8 \\
8 & Retention time, $\mathrm{min}$ & 32.26 \\
9 & LOD $(\mu \mathrm{g} / \mathrm{ml})$ & 0.32 \\
10 & LOQ $(\mu \mathrm{g} / \mathrm{ml})$ & 0.45 \\
\hline
\end{tabular}

RSD, relative standard deviation; AT, Ailanthone.

mobile phase $\mathrm{B}$ and $1 \%$ methanol-water in the mobile phase A, provided a better resolution and separation, and resulted in high precision sensitivity and selectivity. The detection wavelengths at 210, 230, 250 and $280 \mathrm{~nm}$ were selected according to the maximum absorption and full-scan experiment, and compared the peak number and peak resolution. Finally, the wavelength was set at $250 \mathrm{~nm}$. The injection volume was set at $10 \mu \mathrm{l}$, the flow rate was set at $0.8 \mathrm{ml} / \mathrm{min}$ and the column temperature was kept at $25^{\circ} \mathrm{C}$. Representative HPLC chromatogram is shown in Fig. 1.

Analytical method validation. Analytical method validation, which includes the linearity, repeatability, precision, LOD, LOQ, stability, and recovery, was performed according to the guidance of International Union of Pure and Applied Chemistry. The linearity was determined by eight concentration levels of AT ranging from $6.44-825 \mu \mathrm{g} / \mathrm{ml}$. The results showed the calibration curve of AT was $y=2.2 x-20.9$, and exhibited excellent linear regressions with a high determination coefficient $\left(r^{2}=0.9992\right)$. We also found that the calibration range adequately covered the amounts of AT in the investigated samples. The LOD, defined as three times the baseline noise, was $0.32 \mu \mathrm{g} / \mathrm{ml}$. The LOQ, defined as 10 times the baseline noise, was $0.45 \mu \mathrm{g} / \mathrm{ml}$. The results of precision, repeatability, recovery, and stability showed that the relative standard deviation (RSD) values were all $<2.3 \%$. The recovery of the method was in the range of $93-102 \%$, with an $\mathrm{RSD}<2.3 \%$. These results demonstrated that the system and the HPLC method was accurate enough for determination of AT and the AA samples. The data are shown in Table I.

Sample analysis. The newly established analytical method was used to quantify the contents of AT in 10 batches of AA from different provinces in China. The results showed that all the AA samples were rich in AT, however, their contents were significantly different. The average content in 10 batches of AT was $0.613 \mathrm{mg} / \mathrm{g}$, while the total contents of AT in different samples ranged from $0.21-1.78 \mathrm{mg} / \mathrm{g}$. These results suggested remarkable differences between different samples. In the point of origin analysis, Henan province reached the highest level, 


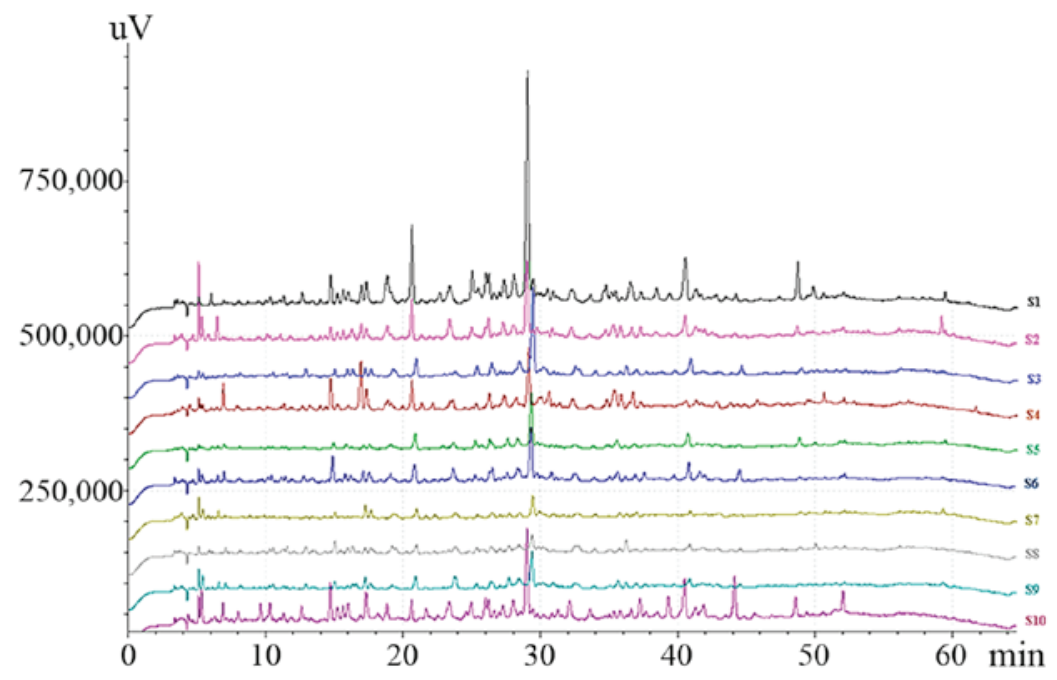

Figure 2. High-performance liquid chromatography (HPLC) fingerprints of 10 batches of Ailanthus altissima (AA).

Table II. Contents of AT in 10 batches of AA samples $(n=3)$.

\begin{tabular}{llccc}
\hline No. & Origin & Batch no. & Content $(\mathrm{mg} / \mathrm{g})$ & $\mathrm{RSD}(\%)$ \\
\hline S1 & Henan & 150317 & 1.78 & 0.13 \\
S2 & Shanxi & 141109 & 0.63 & 0.08 \\
S3 & Anhui & 150502 & 0.75 & 0.11 \\
S4 & Hebei & 150104 & 0.52 & 0.06 \\
S5 & Guizhou & 141208 & 0.48 & 0.15 \\
S6 & Guangxi & 150106 & 0.45 & 0.03 \\
S7 & Sichuan & 150212 & 0.24 & 0.13 \\
S8 & Yunnan & 150411 & 0.21 & 0.08 \\
S9 & Zhejiang & 140219 & 0.35 & 0.12 \\
S10 & Jiangsu & 150101 & 0.72 & 0.08 \\
\hline
\end{tabular}

RSD, relative standard deviation; AT, Ailanthone; AA, Ailanthus altissima.

whereas it was only $0.21 \mathrm{mg} / \mathrm{g}$ in S8, which was from Yunnan province (Table II). These data provide an important reference for the quality of AA used as herbal medicine for the treatment of cancers or as a material to obtain the AT for further use.

HPLC fingerprint of $A A$. Altogether, fingerprints of the 10 batches of samples were analyzed. AT peak which is the main component of AA, was set as the reference peak. The simulated mean chromatogram was generated by the Computer-Aided Similarity Evaluation System (CASE) for Chromatographic Fingerprint of TCM (China Committee of Pharmacopeia, 2008). Nineteen common peaks were selected as characteristic peaks (Fig. 2). The relative retention time of each peak was calculated (Table III). The RSD of the relative retention time of common peaks for all 10 batches was $<1.5 \%$, which is in line with the requirements of fingerprints by the HPLC method.

The State Food and Drug Administration (SFDA) suggested that all herbal chromatograms should be evaluated in terms of similarity by calculating the correlation coefficient and/or angle cosine value of the original data (24-26). In this study,
SA was conducted based on the standard fingerprints, and the results are shown in Table IV. The relative retention time similarities of communal peaks were all $>0.99$, which indicates that different samples had similar constituents. However, peak area similarities of communal peaks were significantly different because of the production process and the contents of main bioactive constituents.

Cell growth inhibition effects of $A A$. In our previous study, we investigated the AA-induced cytotoxicity and apoptosis in hepatoma carcinoma cells. To evaluate the cytotoxicity of AA against brain glioma cells, U87 cell line was treated with 0.1-1 mg/l AA for 1-3 days. The cell viability was measured by using the MTT assay. AA potently inhibited the growth of U87 in a concentration-dependent manner (Fig. 3A). Further, we also found AA inhibited cell proliferation of U87 in a timedependent manner (Fig. 3B). Thus, 0.3, 0.6 and $0.9 \mathrm{mg} / \mathrm{l} \mathrm{AA}$ were selected for further study.

AA induces apoptosis in brain glioma cells. AA caused U87 cell death and, therefore, the role of apoptosis in the process was determined subsequently. The occurrence of apoptosis was performed by an Annexin V-FITC/propidium iodide (PI) double-staining assay. As shown in Fig. 4A, the percentage of apoptotic cells (including early and late apoptotic cells) increased significantly from 5.3 to $56.2 \%$. As caspase activation plays an important role in the process of apoptosis, western blotting was performed to examine caspase activation. As shown in Fig. 4B, cleaved caspase- 8 and caspase-3 were clearly increased in AA-treated cells. To evaluate the role of caspases in cell apoptosis, U87 cells were pretreated with the pan-caspase inhibitor $\mathrm{z}-\mathrm{VAD}$-fmk for $3 \mathrm{~h}$ prior to treatment. As shown in Fig. 4C, z-VAD-fmk pretreatment significantly reduced cell death caused by AA. These results confirmed that AA may promote apoptosis via a caspase-dependent mechanism.

AA induces oxidative stress in brain glioma cells. To further investigate whether oxidative stress was involved in the AA-induced apoptosis, ROS and MDA levels in U87 cells were measured. As shown in Fig. 5A, AA induced the 
Table III. The relative retention time of each peak.

\begin{tabular}{|c|c|c|c|c|c|c|c|c|c|c|c|}
\hline \multirow[b]{2}{*}{ Peak no. } & \multicolumn{11}{|c|}{ Batches } \\
\hline & S1 & S2 & S3 & S4 & S5 & S6 & S7 & S8 & S9 & S10 & $\mathrm{RSD} / \%$ \\
\hline 1 & 0.1765 & 0.1769 & 0.1744 & 0.1761 & 0.1747 & 0.1752 & 0.1748 & 0.1745 & 0.1750 & 0.1769 & 0.57 \\
\hline 2 & 0.2389 & 0.2381 & 0.2404 & 0.2375 & 0.2381 & 0.2380 & 0.2368 & 0.2409 & 0.2409 & 0.2374 & 0.63 \\
\hline 3 & 0.3562 & 0.3554 & 0.3588 & 0.3553 & 0.3572 & 0.3558 & 0.3583 & 0.3593 & 0.3591 & 0.3553 & 0.47 \\
\hline 4 & 0.3901 & 0.4055 & 0.3931 & 0.3892 & 0.3916 & 0.3902 & 0.3930 & 0.3938 & 0.3935 & 0.3899 & 1.20 \\
\hline 5 & 0.5240 & 0.5243 & 0.5112 & 0.5230 & 0.5093 & 0.5074 & 0.5114 & 0.5118 & 0.5117 & 0.5246 & 1.38 \\
\hline 6 & 0.5386 & 0.5393 & 0.5422 & 0.5443 & 0.5408 & 0.5394 & 0.5424 & 0.5434 & 0.5435 & 0.5506 & 0.64 \\
\hline 7 & 0.5966 & 0.5953 & 0.5869 & 0.5931 & 0.5846 & 0.5834 & 0.5870 & 0.5871 & 0.5874 & 0.5966 & 0.86 \\
\hline 8 & 0.7106 & 0.7109 & 0.7122 & 0.7087 & 0.7115 & 0.7108 & 0.7128 & 0.7133 & 0.7119 & 0.7106 & 0.19 \\
\hline 9 & 0.8053 & 0.8061 & 0.8090 & 0.8114 & 0.8068 & 0.8072 & 0.8089 & 0.8109 & 0.8095 & 0.8051 & 0.28 \\
\hline 10 & 0.8617 & 0.8613 & 0.8626 & 0.8694 & 0.8621 & 0.8621 & 0.8634 & 0.8633 & 0.8630 & 0.8623 & 0.27 \\
\hline 11 & 0.9653 & 0.9667 & 0.9674 & 0.9692 & 0.9662 & 0.9667 & 0.9670 & 0.9580 & 0.9673 & 0.9647 & 0.31 \\
\hline 12 & 1.0000 & 1.0000 & 1.0000 & 1.0000 & 1.0000 & 1.0000 & 1.0000 & 1.0000 & 1.0000 & 1.0000 & 0.00 \\
\hline 13 & 1.2096 & 1.2338 & 1.2173 & 1.2312 & 1.2120 & 1.2152 & 1.2152 & 1.2171 & 1.2137 & 1.2334 & 0.76 \\
\hline 14 & 1.2890 & 1.2841 & 1.2817 & 1.2799 & 1.2805 & 1.2813 & 1.2566 & 1.2806 & 1.2811 & 1.2982 & 0.81 \\
\hline 15 & 1.3957 & 1.3961 & 1.3909 & 1.3940 & 1.3892 & 1.3917 & 1.3898 & 1.3903 & 1.3907 & 1.3947 & 0.18 \\
\hline 16 & 1.4346 & 1.4445 & 1.4210 & 1.4316 & 1.4291 & 1.4309 & 1.4271 & 1.4218 & 1.4278 & 1.4426 & 0.54 \\
\hline 17 & 1.5218 & 1.5216 & 1.5170 & 1.5388 & 1.5163 & 1.5175 & 1.5128 & 1.5156 & 1.5157 & 1.5203 & 0.48 \\
\hline 18 & 1.6767 & 1.6770 & 1.6643 & 1.6778 & 1.6652 & 1.6672 & 1.6631 & 1.6639 & 1.6641 & 1.6729 & 0.37 \\
\hline 19 & 2.0456 & 2.0391 & 2.0124 & 2.0290 & 2.0188 & 2.0210 & 2.0145 & 2.0146 & 2.0178 & 2.0478 & 0.66 \\
\hline
\end{tabular}

Table IV. Relative retention time and peak area similarities of AA.

\begin{tabular}{lcccccccccc}
\hline & \multicolumn{10}{c}{ Batches } \\
\cline { 2 - 11 } Items & S1 & S2 & S3 & S4 & S5 & S6 & S7 & S8 & S9 & S10 \\
\hline Relative retention time similarities & 0.999 & 0.999 & 0.998 & 0.998 & 0.999 & 0.999 & 0.998 & 0.999 & 0.999 & 0.999 \\
Peak areas similarities & 0.912 & 0.897 & 0.954 & 0.838 & 0.911 & 0.834 & 0.753 & 0.682 & 0.869 & 0.934 \\
\hline
\end{tabular}

AA, Ailanthus altissima.

production of ROS in U87 cells in a dose-dependent manner. ROS always induce lipid peroxidation of cells, so we also measured the MDA levels. As the results show in Fig. 5B, MDA levels were induced by AA treatment and also in a dose-dependent manner. To evaluate the role of ROS in cell apoptosis, U87 cells were pretreated with the ROS scavenger, NAC. Cotreatment with NAC attenuated both AA-induced apoptosis and growth inhibition in U87 cells (Fig. 5C and D). These results suggested that ROS production occurs upstream of AA-induced apoptosis in U87 cells.

AA induces ER stress in brain glioma cells. To further investigate whether other signaling molecules are involved in the AA-induced apoptosis, ER stress was measured. Our results showed that ER stress makers PERK and elF2 $\alpha$ were phosphorylated by AA treatment (Fig. 6A). To evaluate the role of ER stress in cell apoptosis, 4PBA (10 mM), an ER stress inhibitor was used. Cotreatment with 4PBA inhibited both AA-induced apoptosis and growth inhibition in U87 cells (Fig. 6B and C). To make clear the relationship between ER stress and oxidative stress in the apoptosis process, NAC was used. We found that cotreatment with NAC decreased the phosphorylation of PERK caused by AA (Fig. 6D). These results suggested that AA induced ER stress through oxidative stress.

\section{Discussion}

AA a Chinese herbal medicine, has shown potent activities in inhibiting cancer (21). AT one of the primary active quassinoids in AA, has also been reported to possess some inhibiting properties in various cancers (20). However, no HPLC analytical method which could be used in the routine analysis to improve the quality control has been reported. In this study, a quick and simple HPLC analytical method was build and its pharmacological studies were also studied.

Different HPLC conditions including mobile phase, column and detection wavelength were investigated to select the optimi- 


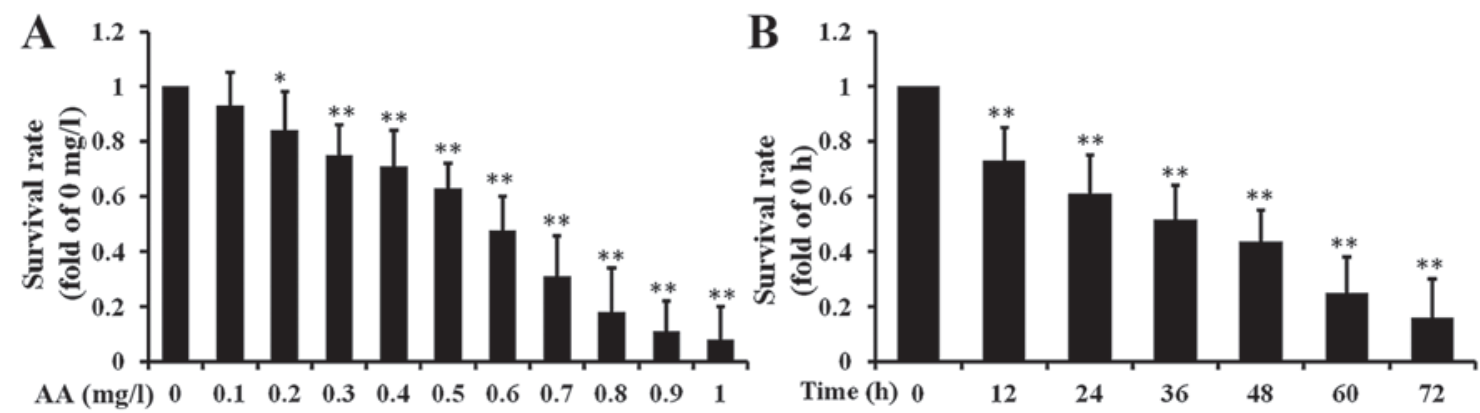

Figure 3. The effects of Ailanthus altissima (AA) on the U87 cell survival rate. (A) U87 cells were treated with various doses (0-1 mg/l) of AA for 24 h, and then cell survival rate was measured by MTT. (B) U87 cells were treated with $0.6 \mathrm{mg} / 1 \mathrm{AA}$ for $0-72 \mathrm{~h}$ and then cell survival rate at every time-point was measured by MTT. Data are expressed as the mean \pm standard deviation of triplicate measurements. ${ }^{*} \mathrm{P}<0.05$ and ${ }^{* *} \mathrm{P}<0.05 \mathrm{vs} .0 \mathrm{mg} / 1 \mathrm{or} 0 \mathrm{~h}$.

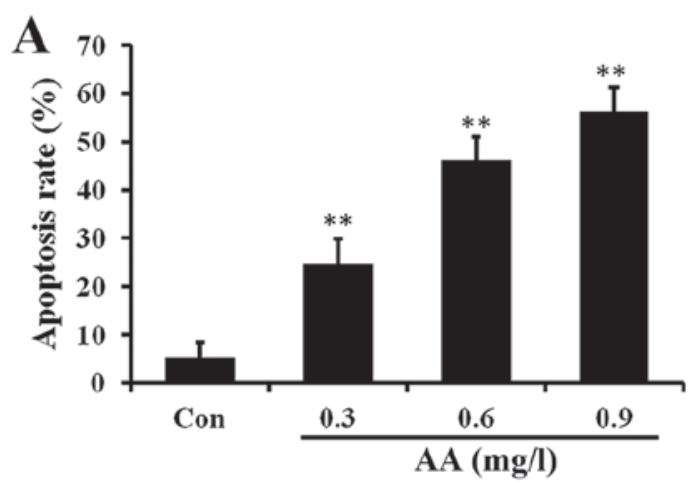

B
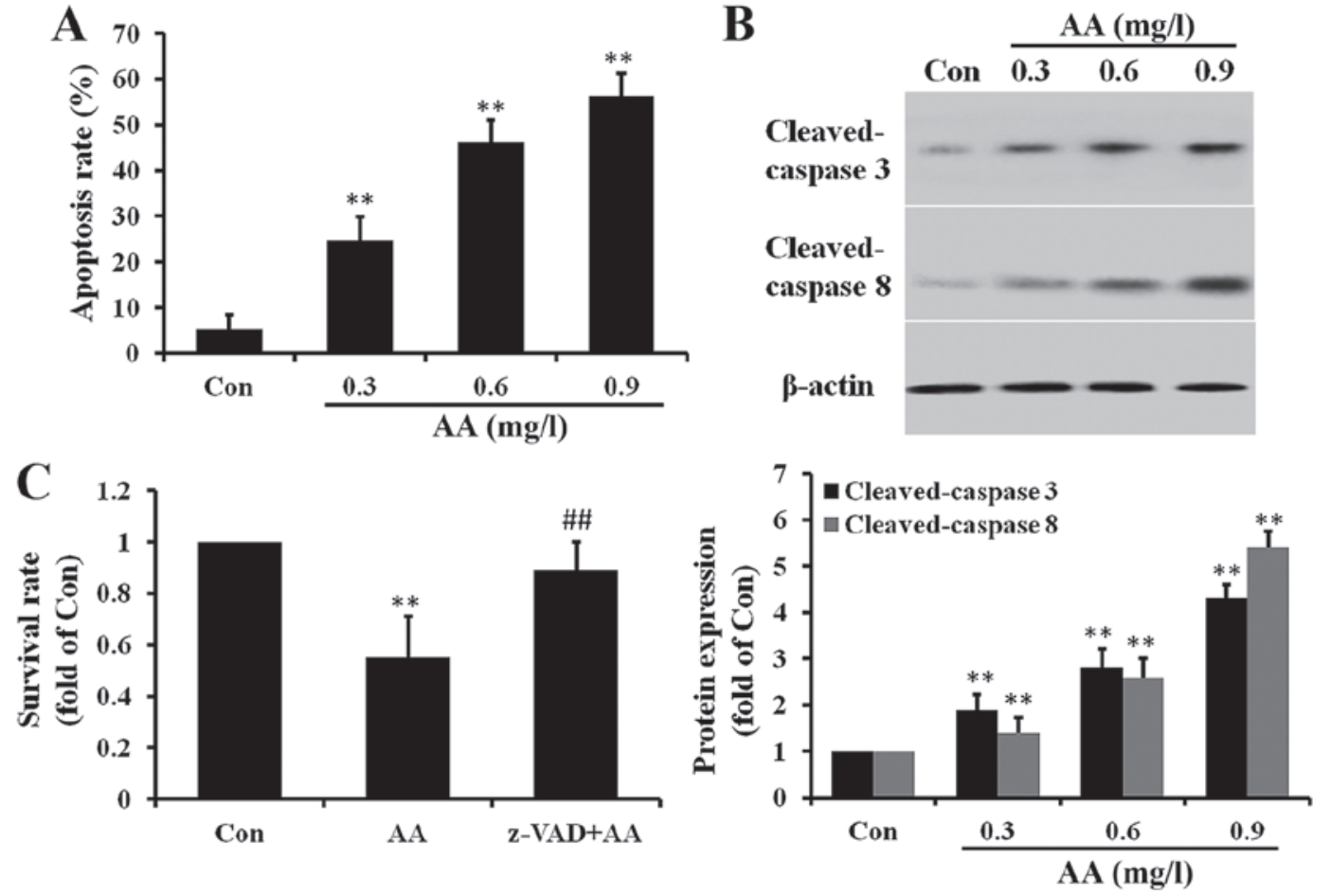

Figure 4. The effects of Ailanthus altissima (AA) on the U87 cell apoptosis. (A) Apoptosis rate was performed by an Annexin V-FITC/propidium iodide (PI) double-staining assay according to the introduction. (B) Expression of cleaved-caspase- 8 and -3 were measured by western blotting. (C) U87 cells were pretreated with the pan-caspase inhibitor z-VAD-fmk $(20 \mu \mathrm{M})$ for $3 \mathrm{~h}$ prior to treatment, and then cell survival rate was measured by MTT. ${ }^{* *} \mathrm{P}<0.01 \mathrm{vs.}$ Con group; ${ }^{\# \#} \mathrm{P}<0.01$ vs. AA treated group.

zation of chromatographic conditions. Three different types of LC columns were investigated: Yilite Hypersil base deactivated silica C18 column, Yilite SinoChrom ODS-BP $\mathrm{C}_{18}$ column, Kromasil 100-5 C18 column. Kromasil 100-5 C18 column were selected because they provided better separation efficiency. The HPLC mobile phase in methanol-water, acetonitrile-water and the different concentrations of modifiers (formic acid, acetic acid, triethylamine and phosphoric acid) were investigated. Finally, $1 \%$ formic acid and $1 \%$ methanol-water was applied for the separation of chemical constituents in AA. The monitoring wavelength was set at $250 \mathrm{~nm}$ for fingerprinting analysis.

Analytical method validation was performed to test the reliability. The linearity of AT was $6.44-825 \mu \mathrm{g} / \mathrm{ml}$, and the determination coefficient was 0.9992 , which indicated the regression model for the calibration curves confirmed the good linearity of the method. The low LOD and LOQ values indicated that the newly established method provides adequate sensitivity. The results of precision, repeatability, recovery, and stability showed that the relative standard deviation (RSD) values were all $<2.3 \%$. These results indicated that the proposed method is well validated and suitable for quantitatively detecting AT in AA.

Toxic, bioactive, characteristic, synergistic, main, correlative and general components were often used as the quality control markers of TCM (27). The chemical marker is useful for those herbal medicines of limited and known chemical composition. In this study, the content of AT was selected as the quality control. Ten batches of AA from different provinces in China were detected, and we found that the contents of AT ranged from $0.21-1.78 \mathrm{mg} / \mathrm{g}$ in different origins. Henan province had the highest level, and Yunnan province had the lowest level. These data provide an important reference for the quality of AA used as herbal medicine. 

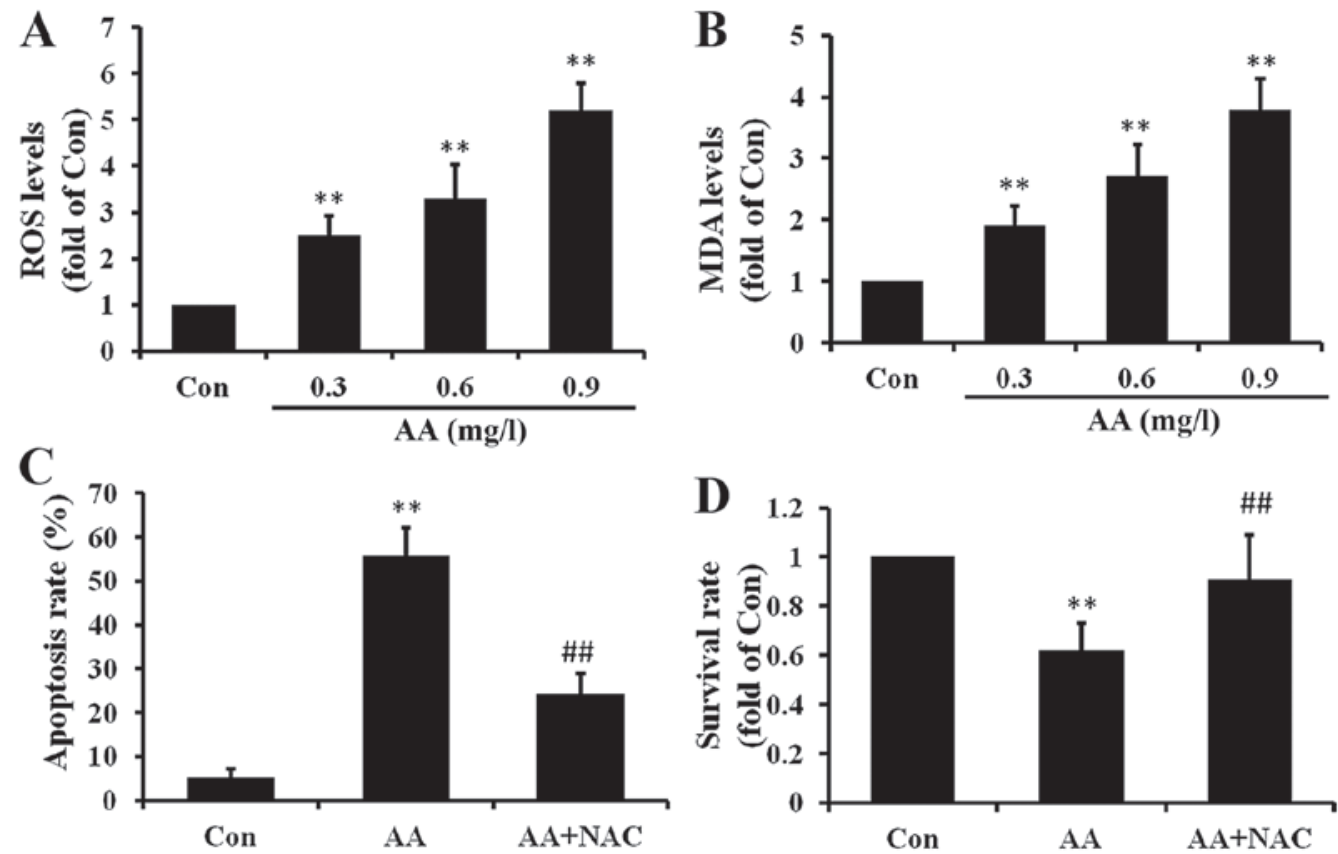

Figure 5. The effects of Ailanthus altissima (AA) on oxidative stress in the U87 cells. (A) ROS levels in cells were measured by reactive oxygen species assay kit (Nanjing Jiancheng) according to the introduction. (B) MDA was measured by malondialdehyde (MDA) assay kit (TBA method) according to the introduction. U87 cells were co-treated with $\mathrm{N}$-acetyl cysteine (NAC, $2.5 \mathrm{mM}$ ) and AA, then apoptosis rate was measured by (C) Annexin V-FITC/propidium iodide (PI) double-staining assay and (D) cell survival rate was measured by MTT. ${ }^{* *} \mathrm{P}<0.01$ vs. Con group; ${ }^{* \#} \mathrm{P}<0.01$ vs. AA treated group.

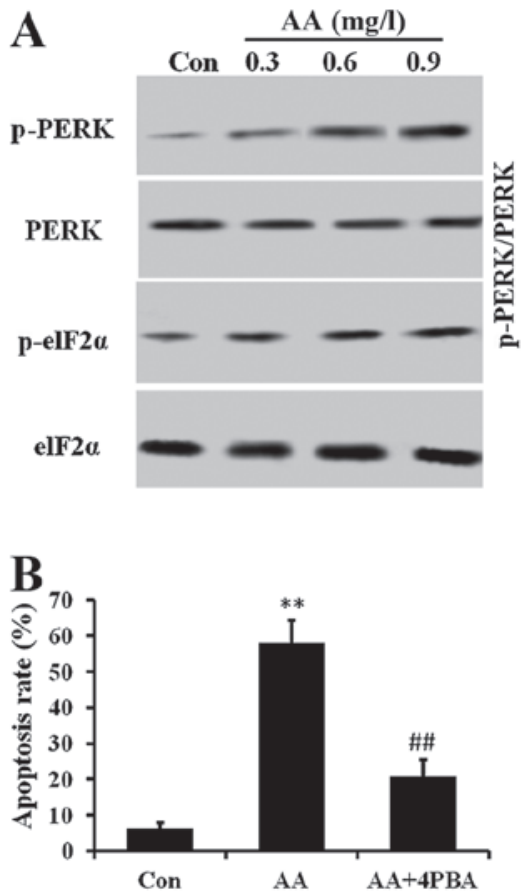

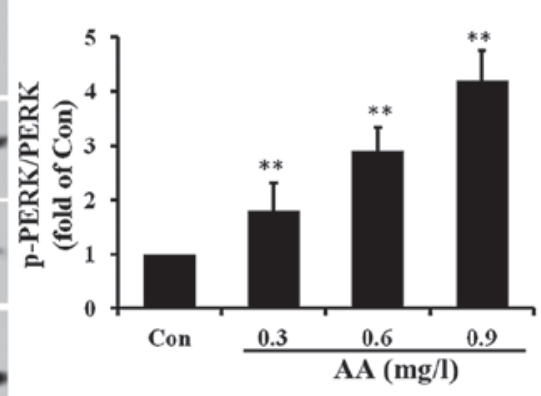

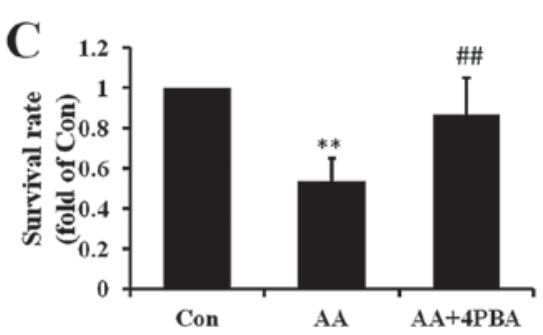

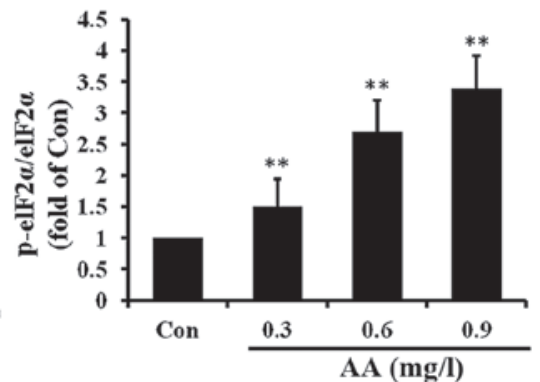

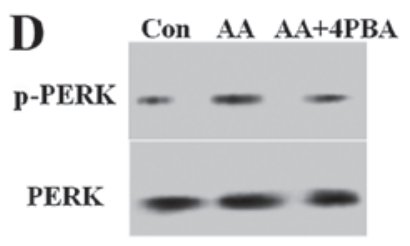

Figure 6. The effects of Ailanthus altissima (AA) on ER stress in the U87 cells. (A) Expression of p-PERK, PERK, elF2 $\alpha$, p-elF2 $\alpha$ were measured by western blotting. (B) U87 cells were co-treated with 4PBA (10 mM), then apoptosis rate was measured by (C) Annexin V-FITC/propidium iodide (PI) double-staining assay and cell survival rate was measured by MTT. (D) U87 cells were co-treated with 4PBA (10 mM), p-PERK and PERK protein levels were measured by western blotting. ${ }^{* *} \mathrm{P}<0.01$ vs. Con group; ${ }^{\# \prime} \mathrm{P}<0.01$ vs. AA treated group.

Chromatographic fingerprinting, a rational and powerful approach to characterize a multi-herb formulation, has been widely used in TCM. There are many analytical methods including X-ray diffraction (XRD), gas chromatography (GC), high-performance liquid chromatography (HPLC), and capillary electrophoresis (CE), used in the fingerprinting analysis (28). HPLC has been proven to be an economical, stable, and reliable method for fingerprinting analysis (29). In this study, 10 batches of AA collected from 10 different regions were analyzed by the HPLC fingerprinting method according to a standard protocol. Nineteen common peaks were selected as characteristic peaks. Results obtained through 
similarity evaluation software showed that the similarity of 10 batches is $<1.5 \%$. The relative retention time similarities of communal peaks were all $>0.99$, and the peak area similarities of communal peaks were significantly different (0.682-0.954). These results demonstrated that different samples had similar constituents and the contents of main bioactive constituents varied in a large range. Therefore, HPLC fingerprinting combined with simultaneous quantification of AT could be taken into the consideration for quality control of AA.

Glioblastoma is the most lethal form of cancer in adults, affecting $>10,000$ patients each year in USA (30). Glioblastoma is characterized by abnormal regulation of glial cell differentiation and its invasiveness and aggressive progression. Once diagnosed with glioblastoma, a median survival is $<2$ years (31). In the past decades, treatment options and medications have remained limited with few advances or success (32). Thus, screening anti-glioblastoma drugs from TCMs may be an effective method. Even through AA has shown good inhibiting activity in treating liver cancer, human breast cancer and cervical cancer, however, the effects of AA on glioblastoma is not known. In this study, U87 cells were used and treated with $\mathrm{AA}$, and then therapeutic effect and action mechanism were investigated. We found that AA inhibited the growth of U87 in a concentration- and time-dependent manner. In addition, we found apoptosis was induced by AA treatment through caspase activation. Oxidative stress and ER stress were also found in AA treated U87 cells, and they all play important roles in apoptosis. The relationships between oxidative stress, ER stress and caspases in cell apoptosis were also investigated by their specific inhibitors. Our results demonstrated that AA induced oxidative stress first in U87 cells, then induced ER stress, finally activated the caspases which caused cell apoptosis.

In conclusion, the results of this study indicated that the combination of chromatographic fingerprint and quantitative analysis could be readily used as a quality control approach for AA. In addition, AA-induced oxidative stress, ER stress and cell apoptosis in U87 cells. Our study demonstrated that AA may be a potential candidate herb for further development as prescription or adjuvant treatment for glioblastoma.

\section{Acknowledgements}

Not applicable.

\section{Funding}

This study was supported by the Shaanxi Science and Technology Innovation Project Plan (no. 2015HBGC-16) and the Key Research Laboratory of Traditional Chinese Medicine and Natural Medicine in Shaanxi Province (no. 2015-164).

\section{Availability of data and material}

The datasets used and/or analyzed during the current study are available from the corresponding author on reasonable request.

\section{Authors' contributions}

QH and SW conceived and designed the study. JL, YL, MJ and FW performed the experiments. HX participated in the work of the HPLC fingerprint analysis of the experiment. YZ and $\mathrm{WW}$ provided the materials. $\mathrm{QH}$ and $\mathrm{SW}$ wrote the paper. JL, YL, MJ and FW reviewed and edited the manuscript. All authors read and approved the manuscript.

\section{Ethics approval and consent to participate}

Not applicable.

\section{Consent for publication}

Not applicable.

\section{Competing interests}

The authors declare that they have no competing interests.

\section{References}

1. Li H, Wang SW, Zhang BL, Xie YH, Yang Q, Cao W and Wang JB: Simultaneous quantitative determination of 9 active components in traditional Chinese medicinal preparation ShuangDan oral liquid by RP-HPLC coupled with photodiode array detection. J Pharm Biomed Anal 56: 820-824, 2011.

2. SW W: Molecular composition theory of Chinese medicinal materials and modern traditional Chinese medicine. J Asia-Pac Tradit 4: 9-12, 2008

3. Xie PS and Leung AY: Understanding the traditional aspect of Chinese medicine in order to achieve meaningful quality control of Chinese materia medica. J Chromatogr A 1216: 1933-1940, 2009.

4. Zhang Z, Li Q, Li Q, Du S, Zhou Y, Lv C, Zhao Y, Wang Y and Zhang N: Simultaneous determination of nineteen major components in Qi She Pill by ultra-high-performance liquid chromatography-tandem mass spectrometry. Acta Pharm Sin B 4: 384-393, 2014.

5. Khan IA: Issues related to botanicals. Life Sci 78: 2033-2038, 2006.

6. Kim HM, Lee JS, Sezirahiga J, Kwon J, Jeong M, Lee D, Choi JH and Jang DS: A new canthinone-type alkaloid isolated from Ailanthus altissima Swingle. Molecules 21: E642, 2016.

7. Howard JL: Ailanthus altissima; Fire Effects Information System, US Department of Agriculture, Forest Service, Rocky Mountain Research Station, Fire Sciences Laboratory: Fort Collins, CO, USA, 20042014.

8. Kowarik I and Saumel I: Biological flora of central Europe: Ailanthus altissima (Mill.) swingle. Perspect Plant Ecol Evol Syst 8: 30, 2007.

9. Jin MH, Yook J, Lee E, Lin CX, Quan Z, Son KH, Bae KH, Kim HP, Kang SS and Chang HW: Anti-inflammatory activity of Ailanthus altissima in ovalbumin-induced lung inflammation. Biol Pharm Bull 29: 884-888, 2006.

10. Kang TH, Choi IY, Kim SJ, Moon PD, Seo JU, Kim JJ, An NH, Kim SH, Kim MH, Um JY, et al: Ailanthus altissima swingle has anti-anaphylactic effect and inhibits inflammatory cytokine expression via suppression of nuclear factor-kappaB activation. In Vitro Cell Dev Biol Anim 46: 72-81, 2010.

11. Casinovi CG, Ceccherelli P, Fardella G and Grandolini G: Isolation and structure of a quassinoid from Ailanthus glandulosa. Phytochemistry 22: 2871-2873, 1983.

12. Kubota K, Fukamiya N, Hamada T, Okano M, Tagahara K and Lee KH: Two new quassinoids, ailantinols A and B, and related compounds from Ailanthus altissima. J Nat Prod 59: 683-686, 1996.

13. Kosuge K, Mitsunaga K, Koike K and Ohmoto T: Studies on the constituents of Ailanthus integrifolia. Chem Pharm Bull (Tokyo) 42: 1669-1671, 1994.

14. Hwang SG, Lee HC, Kim CK, Kim DG, Lee GO, Yun YG, Jeon BH: Effect of Ailanthus altissima water extract on cell cycle control genes in Jurkat T lymphocytes. Yakhak Hoechi 46: 18-23, 2002.

15. Tamura S, Fukamiya N, Mou XY, Mukainaka T, Tokuda H, Nishino H, Tagahara K, Koike K, Lee KH and Okano M: Conversion of quassinoids for enhancement of inhibitory effect against Epstein-Barr virus early antigen activation. Introduction of lipophilic side chain and esterification of diosphenol. Chem Pharm Bull (Tokyo) 48: 876-878, 2000. 
16. Okunade AL, Bikoff RE, Casper SJ, Oksman A, Goldberg DE and Lewis WH: Antiplasmodial activity of extracts and quassinoids isolated from seedlings of Ailanthus altissima (Simaroubaceae). Phytother Res 17: 675-677, 2003.

17. Fukamiya N, Lee KH, Muhammad I, Murakami C, Okano M, Harvey I and Pelletier J: Structure-activity relationships of quassinoids for eukaryotic protein synthesis. Cancer Lett 220 $37-48,2005$.

18. Rosati A, Quaranta E, Ammirante M, Turco MC, Leone A and De Feo V: Quassinoids can induce mitochondrial membrane depolarisation and caspase 3 activation in human cells. Cell Death Differ 11 (Suppl 2): S216-S218, 2004.

19. Wang Y, Wang WJ, Su C, Zhang DM, Xu LP, He RR, Wang L, Zhang J, Zhang XQ and Ye WC: Cytotoxic quassinoids from Ailanthus altissima. Bioorg Med Chem Lett 23: 654-657, 2013.

20. Yang XL, Yuan YL,Zhang DM, Li F and Ye WC: Shinjulactone O, a new quassinoid from the root bark of Ailanthus altissima. Nat Prod Res 28: 1432-1437, 2014.

21. Ishibashi M, Tsuyuki T and Takahashi T: Bitter principles of Ailanthus altissima SWINGLE. Conversion of ailanthone into shinjulactone C. Bull Chem Soc Jpn 58: 2357-2360, 1985.

22. Jaziri M, Diallo B and Vanhaelen M: Separation of quassinoids from Ailanthus altissima by high-speed counter-current chromatography. J Chromatogr A 538: 227-229, 1991.

23. Furey A, Moriarty M, Bane V, Kinsella B and Lehane M: Ion suppression; a critical review on causes, evaluation, prevention and applications. Talanta 115: 104-122, 2013.

24. Jin XF, Lu YH, Wei DZ and Wang ZT: Chemical fingerprint and quantitative analysis of Salvia plebeia R.Br. by high-performance liquid chromatography. J Pharm Biomed Anal 48: 100-104, 2008.

25. Tang D, Yang D, Tang A, Gao Y, Jiang X, Mou J and Yin X: Simultaneous chemical fingerprint and quantitative analysis of Ginkgo biloba extract by HPLC-DAD. Anal Bioanal Chem 396: 3087-3095, 2010.
26. Wei H, Sun L, Tai Z, Gao S, Xu W and Chen W: A simple and sensitive HPLC method for the simultaneous determination of eight bioactive components and fingerprint analysis of Schisandra sphenanthera. Anal Chim Acta 662: 97-104, 2010.

27. Liu EH, Qi LW, Li K, Chu C and Li P: Recent advances in quality control of traditional Chinese medicines. Comb Chem High Throughput Screen 13: 869-884, 2010.

28. Zhong XK, Li DC and Jiang JG: Identification and quality control of Chinese medicine based on the fingerprint techniques. Curr Med Chem 16: 3064-3075, 2009.

29. Li T, Zhuang S, Wang Y, Wang Y, Wang W, Zhang H, Chen L, Wang D, Zhou Z and Yang W: Flavonoid profiling of a traditional Chinese medicine formula of Huangqin Tang using high performance liquid chromatography. Acta Pharm Sin B 6: 148-157, 2016.

30. Ostrom QT, Gittleman H, de Blank PM, Finlay JL, Gurney JG, McKean-Cowdin R, Stearns DS, Wolff JE, Liu M, Wolinsky Y, et al: American brain tumor association adolescent and young adult primary brain and central nervous system tumors diagnosed in the United States in 2008-2012. Neuro Oncol 18 (Suppl 1): i1-i50, 2016.

31. Adamson C, Kanu OO, Mehta AI, Di C, Lin N, Mattox AK and Bigner DD: Glioblastoma multiforme: A review of where we have been and where we are going. Expert Opin Investig Drugs 18: 1061-1083, 2009

32. Levin VA, Tonge PJ, Gallo JM, Birtwistle MR, Dar AC, Iavarone A, Paddison PJ, Heffron TP, Elmquist WF, Lachowicz JE, et al: CNS anticancer drug discovery and development conference white paper. Neuro Oncol 17 (Suppl 6): vi1-vi26, 2015. 